\title{
THE CLASS OF EVOLUTIONARY FERRODYNAMIC EQUATIONS
}

\author{
Yuri VIRCHENKO ${ }^{1}$ and Andrey Subbotin ${ }^{1}$ \\ ${ }^{1}$ Belgorod National Research University
}

May 5, 2020

\begin{abstract}
A linear manifold $\$\{\backslash$ mathcal $\mathrm{K}\} \_2 \$$ of evolutionary equations for a pseudovector field on $\$\{\backslash \mathrm{Bbb} \mathrm{R}\}^{\wedge} 3 \$$ is described. An infinitisimal shift of each equation is determined by a second-order differential operator of divergent type. All operators are invariant with respect to space translations in $\$\{\backslash \mathrm{Bbb} R\}^{\wedge} 3 \$$, relative to time translations, and they are transformed by covariant way relative to rotations of $\$\{\backslash \mathrm{Bbb} R\}^{\wedge} 3 \$$. It is proved that the linear space $\$\{\backslash \text { mathcal } \mathrm{M}\}_{-} 2 \backslash$ subset $\{\backslash$ mathcal $\mathrm{K}\} \_2 \$$ of differential operators preserving solenoidal property and unimodularity of the field is one-dimensional and an explicit form of such operators is found.
\end{abstract}

\section{Hosted file}

AppliedMathematics.pdf available at https://authorea.com/users/292892/articles/420732-theclass-of-evolutionary-ferrodynamic-equations 